\title{
Pulsed-laser induced desorption and subsequent readsorption in ambient gas
}

\author{
Gregor Holler, Uwe Albrecht, Stephan Herminghaus, ${ }^{\text {a) }}$ and Paul Leiderer \\ Fakultät Für Physik, Universität Konstanz, D-7750 Konstanz, Germany
}

(Received 3 February 1993; accepted for publication 29 March 1993)

\begin{abstract}
We have investigated the phenomenon of readsorption after pulsed-laser induced thermal desorption during the presence of ambient gas. The temporal evolution of the readsorption of water has been measured as a function of the ambient gas pressure for helium and carbon-dioxide atmospheres. $\mathrm{CO}_{2}$ pressures above $10 \mathrm{mbar}$ lead to a virtually complete readsorption of the desorbed material. A model based on a nearly one-dimensional diffusion scheme explains this remarkable result.
\end{abstract}

Many annealing procedures at surfaces rely on an efficient desorption of molecules or atoms from that surface. This is the case for some slow thermal annealing processes, where e.g., selective desorption from protrusions renders the surface smoother, ${ }^{1}$ as well as for a number of fast pulsed-laser induced annealing techniques, where surface contaminants are desorbed. ${ }^{2}$ Some of these procedures cannot easily be performed in vacuum, most of them would be at least greatly simplified if performed under normal atmosphere. We thus have investigated how the efficiency of pulsed-laser induced thermal desorption is affected by the presence of a gas atmosphere adjacent to the surface under consideration. It turns out that already at moderate pressures (a few mbar) virtually all of the desorbed material finally recondenses on the sample within less than a millisecond. We show that this remarkable result is closely related to the mathematical fact that the recurrence probability of a one-dimensional random walk is unity.

Thin water films adsorbed on a silver surface were used as a model system. The surface under inspection was mounted on a cooling stage in an optically accessible chamber which could be evacuated and filled with a gas (we have used ${ }^{4} \mathrm{He}$ and $\mathrm{CO}_{2}$ as ambient gases) at a controlled pressure. Adjusting the surface temperature relative to a water reservoir within the chamber controlled the thickness of the adsorbed water film. We have employed a ruby laser $(\lambda=694 \mathrm{~nm}$, pulse width $20 \mathrm{~ns})$ to desorb the film. The pulse energy was typically around $2 \mathrm{~mJ}$ at a laser spot diameter of $\sim 5 \mathrm{~mm}$, leading to a temperature rise of some $100 \mathrm{~K},{ }^{3}$ sufficient for the desorption of a substantial fraction of the water film. After the pulse the surface cools back to room temperature within less than $1 \mu \mathrm{s}$. ${ }^{4}$

The water film thickness before and after the pulsedlaser induced desorption was accurately monitored with high temporal resolution by means of optically excited surface plasmons using the Kretschmann configuration. ${ }^{5}$ Surface plasmons were optically excited on a silver film which had been deposited by thermal evaporation on the base of a glass prism. Excitation of the plasmons then shows up in a strong attenuation of the totally reflected light intensity at the resonance angle. This angle is influenced by the thickness of a thin film on the silver surface. The water film

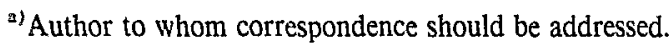

thickness could then be inferred from the reflected light intensity in the slope of the resonance minimum corresponding to the excitation of the surface plasmon which was recorded by means of a fast photodiode (details of the technique are given elsewhere, see Refs. 6,7). The spot of the test laser $(\lambda=633 \mathrm{~nm})$ was centered at the pulse-laser spot and had less than half its diameter, i.e., the measured signal originates exclusively in the region where desorption takes place.

Figure 1 shows traces of water film thickness vs time for laser-induced desorption events at two different $\mathrm{CO}_{2}$ pressures $p_{\mathrm{CO}_{2}}$ in the experimental cell. A desorption of about $6 \AA$ of water is observed at the instant of the laser pulse $(t=0)$, followed by a readsorption of a fraction of the initially desorbed molecules. The extent of the readsorption apparently depends on the ambient gas pressure, being more pronounced for higher pressures. All this occurs on a time scale of about $100 \mu \mathrm{s}$, orders of magnitude faster than the times necessary to reestablish the thermodynamic equilibrium film thickness, being on the order of a few minutes. Figure 2 shows the readsorbed fraction of the desorbed film as a function of ambient gas pressure for $\mathrm{CO}_{2}$ and helium as ambient gases. Obviously, readsorption is larger for the heavier $\mathrm{CO}_{2}$ molecules above the surface.

This readsorption behavior can be explained by diffusion of the desorbed molecules in the ambient gas. If the

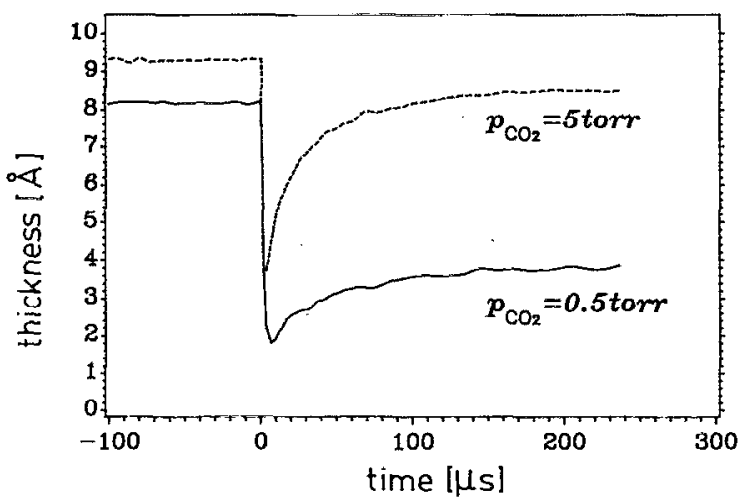

FIG. 1. Water film thickness vs time during a pulse-laser induced desorption experiment ( $t=0$ is set to the instant of the laser pulse) under ambient $\mathrm{CO}_{2}^{-}$gas. Readsorption occurs within about $100 \mu$ s after desorption and is more pronounced for higher gas pressures $p_{\mathrm{CO}}$. 

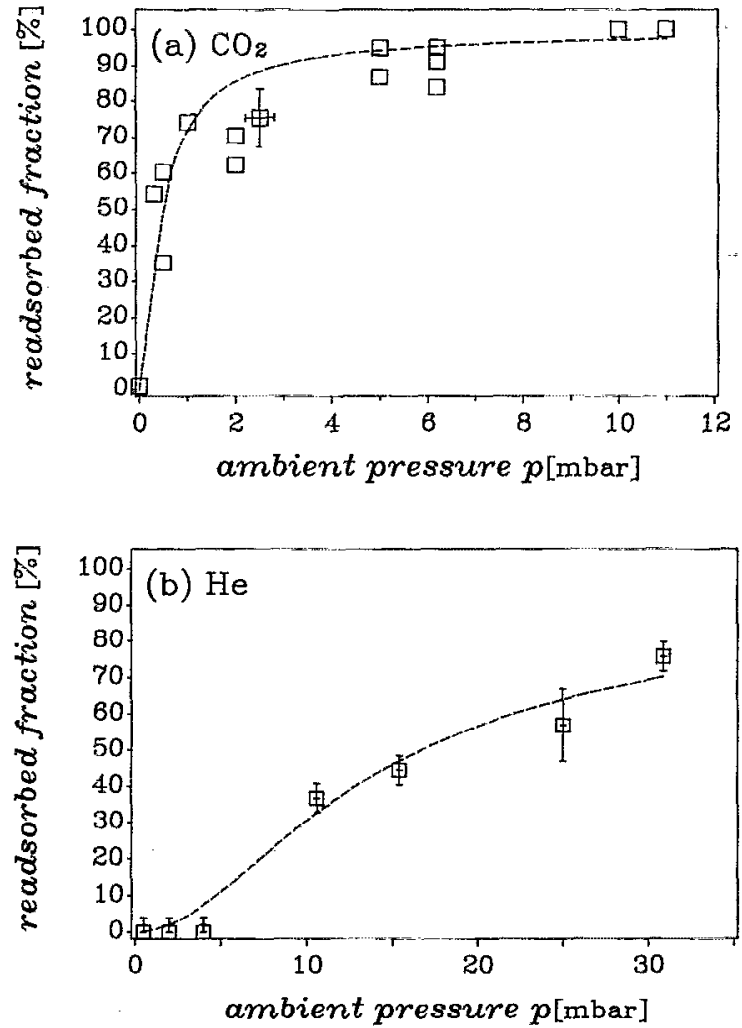

FIG. 2. Readsorbed fraction vs ambient gas pressure for (a) $\mathrm{CO}_{2}$ and (b) ${ }^{4} \mathrm{He}$ gas in the cxperimental cell. The dotted lincs show the predictions of the model described in the text.

diameter of the pulsed-laser spot were infinite, the distribution function of the molecules would be translationally invariant parallel to the surface. Consequently, the diffusion would be equivalent to a one-dimensional random walk with one trapping site representing the sample surface. In this case, all of the initially desorbed material will eventually recondense on the surface, since the recurrence probability in one-dimension is equal to unity. ${ }^{8}$

In the experiment, the diameter of the laser spot is finite, and some of the material will consequently readsorb outside the spot. This reduces the amount of readsorbed material in the center of the spot. The dynamics of the whole process is governed by the diffusion equation

$$
\left[D\left(\partial_{x}^{2}+\partial_{y}^{2}+\partial_{z}^{2}\right)-\partial_{t}\right] \rho(x, y, z, t)=0,
$$

$D$ is the diffusion coefficient and $\rho$ is the number density of the molecules diffusing in the surrounding gas. The sample surface is located in the plane $z=0$. As the initial condition, we assume for the sake of simplicity that all of the desorbed molecules start diffusing at a distance $w$ from the surface, which we call the thermalization length. This length is approximately equal to the mean free path when the masses of the desorbed and the ambient gas molecules are equal, but is expected to be greater than that when the desorbed molecules are heavier than those of the surrounding gas.

In order to account for the fact that there is a "trap" at the locus of the surface, we require $\rho(-z)=-\rho(z)$. The amount of material readsorbed on the surface is given by

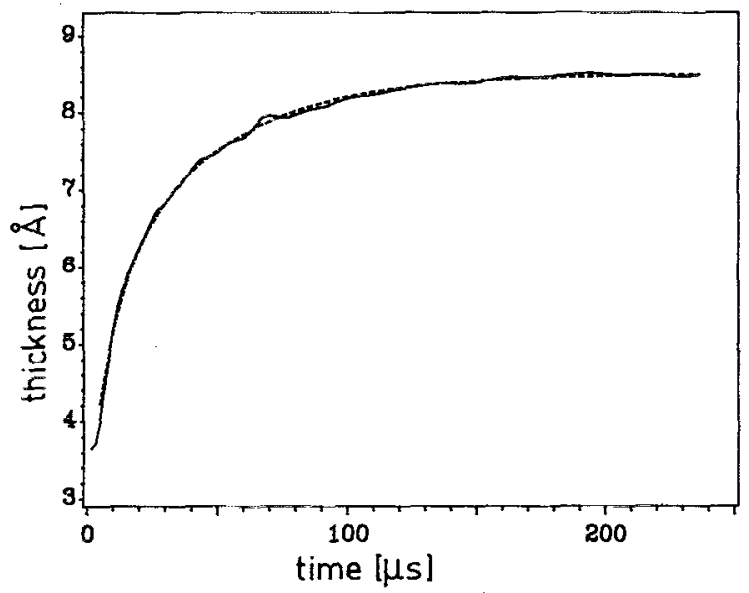

FIG. 3. Temporal evolution of the readsorption for ambient $\mathrm{CO}_{2}$ at a pressure of $5 \mathrm{mbar}$. The dotted line shows an approximate of the timedependent solution of the diffusion equation in excellent agreement with the data.

$$
S(x, y, t)=D \int_{0}^{t} \partial_{z} \rho\left(x, y, z, t^{\prime}\right) d t^{\prime} .
$$

Fourier expanding $\rho$ in all three space coordinates and inserting it into Eq. (1), we obtain

$$
S(x, y, t)=f(\mathbf{r})\left[\frac{2 w}{\left(w^{2}+r^{2}\right)^{3 / 2}}\left(\xi e^{-\xi^{2}}+\operatorname{erfc}(\xi)\right)\right] .
$$

Here, $\mathbf{r}$ is the vector $(x, y), f(\mathbf{r})$ is the number density of molecules desorbed at $\mathbf{r}, \xi$ is defined as $\sqrt{\left(w^{2}+r^{2}\right) / 4 D t}$, and $\circ$ denotes the convolution with respect to r. For $t \rightarrow \infty$, this yields in the case of uniform desorption within a pulsed laser spot of radius $R$ (i.e., $f(\mathbf{r})=\left(\pi R^{2}\right)^{-1}$ for $\mathbf{r}^{2} \leqslant R^{2}$ and zero elsewhere)

$$
S(0,0, \infty)=1-\left(1+\frac{R^{2}}{w^{2}}\right)^{-1 / 2}
$$

This function is plotted as the dashed line in Fig. 2. The experimental features are well reproduced. The thermalization length $w$ is the only fit parameter, the fitted values are consistent with the corresponding estimates for the experimental conditions.

Even better agreement is found for the readsorption transients. The dashed line in Fig. 3 represents a solution of Eq. (2) in the limit of short mean free path compared to the laser spot diameter, allowing a one-dimensional treatment of the diffusion where the time-dependent solution can be expressed as an infinite series. This approximation applies to the measurements at higher ambient gas pressures like the data for $p_{\mathrm{CO}_{2}} 5 \mathrm{mbar}$ shown in Fig. 3. The calculated readsorption transient very closely follows the measured curve. This result once more indicates that the observed readsorption behavior is a consequence of the diffusive scattering of the desorbed molecules in the surrounding gas atmosphere.

In conclusion, we have shown that molecules which are pulsed-laser desorbed from a surface can readsorb within a few $100 \mu$ s due to scattering from ambient gas atoms. The readsorbed fraction increases with molecular 
weight and pressure of the ambient gas, and is substantial already at pressures of a few mbar. The essential features of the observed behavior are well described by the scattering and subsequent diffusion of the desorbed molecules in the ambient gas. The results demonstrate that the presence of a gas atmosphere drastically reduces the efficiency of pulsed laser desorption and should necessarily be taken into account in corresponding experiments.

This work was supported by the SFB 306 of the Deutsche Forschungsgemeinschaft.
'U. Albrecht, K. Kono, and P. Leiderer, Surf. Sci. 283, 419 (1993).

${ }^{2}$ W. Zapka, W. Ziemlich, and A. C. Tam, Appl. Phys. Lett. 58, 2217 (1991).

${ }^{3}$ In order to achieve an efficient coupling of the laser energy into the silver surface, the laser illuminated the surface at the surface plasmon resonance angle.

${ }^{4}$ S. Herminghaus and P. Leiderer, Appl. Phys. A 51, 350 (1990).

${ }^{5}$ E. Kretschmann and H. Raether, Z. Naturforsch. A 23, 2135 (1968).

${ }^{6}$ S. Herminghaus and P. Leiderer, Appl. Phys. Lett. 58, 352 (1991).

${ }^{7}$ S. Herminghaus and P. Leiderer, Proc. SPIE 1594, 334 (1992).

${ }^{8}$ H. Kehr, Phys. Rep. 150, 263 (1987). 